\title{
Microwave-Promoted and Chelation-Controlled Double Arylations of Terminal Olefinic Carbon of Vinyl Ethers
}

\author{
Andreas Svennebring, Peter Nilsson, Mats Larhed*
}

Organic Pharmaceutical Chemistry, Department of Medicinal Chemistry, Uppsala Biomedical Centre, Uppsala University, Box 574, SE-751 23 Uppsala, Sweden.

mats@orgfarm.uu.se

\section{Supporting Information}

Procedures

Materials

$N, N$-Dimethyl-2-[(2,2-di-o-methylphenyl)ethenyloxy]ethanamine (4b)

$N, N$-Dimethyl-2-[(2,2-di-p-chlorobenzene)ethenyloxy]ethanamine (4d) S3

$N, N$-Dimethyl-2-[(2,2-di-p-formylphenyl)ethenyloxy]ethanamine (4e)

Procedure for Preparation of 5 a

Procedure for Preparation of $5 \mathbf{b}$

$N, N$-Dimethyl-2-[(2-phenyl-2-(4- $N, N$-dimetylaminophenyl))ethenyloxy] ethanamine (6a)

$N, N$-Dimethyl-2-[(2-phenyl-2-(2,6-dimethylphenyl))ethenyloxy]ethanamine (6b)

(E)-N,N-Dimethyl-2-[(2-phenyl-1-(2,6-dimethylphenyl))ethenyloxy]ethanamine

$N, N$-Dimethyl-2-[(2-phenyl-2-(o-cyanophenyl))ethenyloxy]ethanamine (6e)

$N, N$-Dimethyl-2-[(2-phenyl-2-(3-tienyl))ethenyloxy]ethanamine (6f)

Procedure for Catalyst Evaluation in the Synthesis of Diarylated Products 
Procedures. ${ }^{1} \mathrm{H}$ and ${ }^{13} \mathrm{C} \mathrm{NMR}$ spectra were obtained in $\mathrm{CDCl}_{3}$ solution at $400 \mathrm{MHz}$ and $100 \mathrm{MHz}$ respectively. All NOESY spectra were obtained in deuterated benzene or chloroform using a $400 \mathrm{MHz}$ spectrometer. Overlapping signals in spectra were assigned as multiplets. Low resolution mass spectra were recorded on a GC-MS instrument equipped with a CP-SIL 8 CB capillary column $(30 \mathrm{~m} \times 0.25$ $\mathrm{mm}$ ) operating at an ionization potential of $70 \mathrm{eV}$. The oven temperature was generally $40-300{ }^{\circ} \mathrm{C}$ (gradient $30{ }^{\circ} \mathrm{C} / \mathrm{min}$ ) although other temperature programs were used to determine $\alpha, \beta / \beta, \beta$ - and $E / Z$ ratios of diarylated products. Regioisomers were assumed to have the same (GC-MS) response factor. All Heck reactions were performed in septa-sealed process vials under air atmosphere, except for the synthesis of $\mathbf{5 a}$ which was conducted as described in the Supporting Information. Microwave heating was carried out using an automatic single-mode synthesizer producing a radiation frequency of 2450 MHz. Silica gel 60 (0.040-0.063 mm, E. Merck, no. 9385) was used for column chromatography.

Materials. Trans-di( $\mu$-aceto)bis[ $o$-(di-o-tolylphosphino)benzyl $]$ dipalladium (II) 3 were obtained from Strem and used as supplied. All other reagents obtained from commercial sources were used as received. The olefin (2-ethenyloxyetyl)dimethylamine (1) was prepared according to literature ${ }^{1}$ or obtained from Aldrich. Compounds, $N, N$-dimethyl-2-[(2,2-diphenyl)ethenyloxy]ethanamine $4 \mathbf{4},{ }^{1} N, N$ dimethyl-2-[(2-phenyl)ethenyloxy]ethanamine $\mathbf{5 a},{ }^{2} \quad N, N$-dimethyl-2-[(2-(2naphthyl))ethenyloxy]ethanamine

$5 \mathbf{b}$ $N, N$-dimethyl-2-[(2-phenyl-2-(2methylphenyl))ethenyloxy]ethanamine $\mathbf{6 c},{ }^{1}$ are known structures. Reported elemental analysis and spectral data were in agreement with the proposed structures. Synthesized compounds lacking elemental analysis in the literature exhibited spectral and analytical properties as summarized below. Compounds $\mathbf{2 a - j}$ and $\mathbf{3}$ are commercially available.

\section{$N, N$-Dimethyl-2-[(2,2-di-o-methylphenyl)ethenyloxy]ethanamine (4b)}

Colorless oil, $80 \%$ yield $\left(0.154 \mathrm{~g}\right.$, reaction at $160{ }^{\circ} \mathrm{C}, 55 \mathrm{~min},>95 \%$ by GC-MS). ${ }^{1} \mathrm{H}$ NMR $(400 \mathrm{MHz}$, $\left.\mathrm{CDCl}_{3}\right) \delta 7.30-7.12(\mathrm{~m}, 8 \mathrm{H}), 6.42(\mathrm{~s}, 1 \mathrm{H}), 4.04(\mathrm{t}, J=6.2 \mathrm{~Hz}, 2 \mathrm{H}), 2.65(\mathrm{t}, J=6.2 \mathrm{~Hz}, 2 \mathrm{H}), 2.33(\mathrm{~s}$, 
3H), $2.31(\mathrm{~s}, 6 \mathrm{H}), 2.13(3 \mathrm{H}) .{ }^{13} \mathrm{C} \mathrm{NMR}\left(100 \mathrm{MHz}, \mathrm{CDCl}_{3}\right) \delta 146.1,140.3,137.7,137.2,137.0,130.9$, $130.8,130.6,130.3,126.9,126.8,125.9,125.3,119.6,71.4,58.9,46.3,20.8 . \mathrm{MS} \mathrm{m} / \mathrm{z}$ (relative intensity $70 \mathrm{eV}) 295\left(\mathrm{M}^{+}, 5\right), 72$ (100), 58 (78). Anal. Calcd for $\mathrm{C}_{20} \mathrm{H}_{25} \mathrm{NO}$ : C, 81.31; H, 8.53. Found: C, 81.3; H, 8.8.

\section{$N, N$-Dimethyl-2-[(2,2-di-p-chlorophenyl)ethenyloxy]ethanamine (4d)}

Yellowish oil, $59 \%$ yield $\left(0.129 \mathrm{~g}\right.$, reaction at $160{ }^{\circ} \mathrm{C}, 55 \mathrm{~min},>95 \%$ by GC-MS). ${ }^{1} \mathrm{H}$ NMR $(400 \mathrm{MHz}$, $\left.\mathrm{CDCl}_{3}\right) \delta 7.24(\mathrm{dt}, J=8.6 \mathrm{~Hz}, 2 \mathrm{H}), 7.22-7.17(\mathrm{~m}, 4 \mathrm{H}), 7.05(\mathrm{dt}, J=8.6 \mathrm{~Hz}, 2 \mathrm{H}), 6.49(\mathrm{~s}, 1 \mathrm{H}), 3.96(\mathrm{t}, J$ $=5.9 \mathrm{~Hz}, 2 \mathrm{H}), 2.56(\mathrm{t}, J=5.9 \mathrm{~Hz}, 2 \mathrm{H}), 2.22(\mathrm{~s}, 6 \mathrm{H}) .{ }^{13} \mathrm{C} \mathrm{NMR}\left(100 \mathrm{MHz}, \mathrm{CDCl}_{3}\right) \delta 146.1,138.9$, 136.0, 132.7, 132.4, 131.4, 129.8, 128.8, 128.4, 118.7, 72.3, 58.9, 46.3. MS m/z (relative intensity 70 eV) 337 (8), 336 (7), $335\left(\mathrm{M}^{+}, 11\right), 72$ (100), 58 (61). Anal. Calcd for $\mathrm{C}_{18} \mathrm{H}_{19} \mathrm{Cl}_{2} \mathrm{NO}$ : C, 64.29; H, 5.70. Found: C, 64.08; H, 5.81.

\section{$N, N$-Dimethyl-2-[(2,2-di-p-formylphenyl)ethenyloxy]ethanamine (4e)}

Colorless oil, $36 \%$ yield $\left(0.076 \mathrm{~g}\right.$, reaction at $180{ }^{\circ} \mathrm{C}, 55 \mathrm{~min},>95 \%$ by GC-MS) was obtained after chromatography using isohexane/chloroform with $1 \% \mathrm{Et}_{3} \mathrm{~N}$ added. FTIR (thin film): $1700 \mathrm{~cm}^{-1} .{ }^{1} \mathrm{H}$ $\operatorname{NMR}\left(400 \mathrm{MHz}, \mathrm{CDCl}_{3}\right) \delta 9.94(\mathrm{~s}, 1 \mathrm{H}), 9.93(\mathrm{~s}, 1 \mathrm{H}), 7.81(\mathrm{~d}, J=8.1 \mathrm{~Hz}, 2 \mathrm{H}), 7.78(\mathrm{~d}, J=8.4 \mathrm{~Hz}$, 2H), $7.52(\mathrm{~d}, J=8.4 \mathrm{~Hz}, 2 \mathrm{H}), 7.31(\mathrm{~d}, J=8.1 \mathrm{~Hz}, 2 \mathrm{H}), 6.77(\mathrm{~s}, 1 \mathrm{H}), 4.11(\mathrm{t}, J=5.9 \mathrm{~Hz}, 2 \mathrm{H}), 2.63(\mathrm{t}, J$ $=5.9 \mathrm{~Hz}, 2 \mathrm{H}), 2.27(\mathrm{~s}, 6 \mathrm{H}) \cdot{ }^{13} \mathrm{C} \mathrm{NMR}\left(100 \mathrm{MHz}, \mathrm{CDCl}_{3}\right) \delta 191.9,191.8,148.8,146.3,143.5,134.82$, 134.77, 130.5, 130.1, 129.6, 128.5, 119.0, 72.3, 58.6, 45.9. MS m/z (relative intensity $70 \mathrm{eV}) 323\left(\mathrm{M}^{+}\right.$, 13), 72 (49), 58 (100). Anal. Calcd for $\mathrm{C}_{20} \mathrm{H}_{21} \mathrm{NO}_{3}$ : C, 74.28; H, 6.55. Found: C, 74.09; H, 6.50.

\section{Procedure for Preparation of $5 \mathbf{a}^{2}$}

Crude compound 5a was prepared according to literature starting from $10.2 \mathrm{~g}(0.050 \mathrm{~mol})$ phenyl iodide. ${ }^{1}$ In order to remove traces of $\alpha$-arylated compound the crude product was extracted three times with $0.1 \mathrm{M} \mathrm{HCl}$. The combined acidic phases was then neutralized with $2 \mathrm{M} \mathrm{NaOH}$ and extracted three 
times with diethyl ether, dried with $\mathrm{K}_{2} \mathrm{CO}_{3}(\mathrm{~s})$ and distilled using a bulb-to-bulb distillation (20 mbar, $\left.140{ }^{\circ} \mathrm{C}\right)$ to afford $87 \%$ yield $(8.31 \mathrm{~g}, 0.044 \mathrm{mmol})$ of pure $\mathbf{5 a}$.

\section{Procedure for Preparation of $\mathbf{5} \mathbf{b}^{3}$}

The following chemicals were added to a thick-walled tube: $3(6.4 \mu \mathrm{mol}, 6.0 \mathrm{mg}), \mathrm{LiCl}(2.64 \mathrm{mmol}$, $112.0 \mathrm{mg}), \mathrm{NaOAc}(1.76 \mathrm{mmol}, 144.4 \mathrm{mg}), \mathrm{K}_{2} \mathrm{CO}_{3}(1.56 \mathrm{mmol}, 216 \mathrm{mg}), 2$-naphthyl bromide (2h, $1.30 \mathrm{mmol}, 269 \mathrm{mg}), 1$ (1.95 mmol, $225 \mathrm{mg})$, DMF (4 mL), water (0.40 mL). The tube was then closed under air and the contents were magnetically stirred and microwave heated at $180{ }^{\circ} \mathrm{C}$ for 15 minutes. After cooling, the reaction mixture was diluted with diethyl ether and extracted three times with $0.1 \mathrm{M}$ $\mathrm{HCl}$. The combined acidic phases were slowly neutralized with $2 \mathrm{M} \mathrm{NaOH}$ and extracted twice with diethyl ether. The etheral phases were combined and dried with $\mathrm{K}_{2} \mathrm{CO}_{3}$ (s). After evaporation of the solvent the compound was purified by bulb-to-bulb distillation $\left(20\right.$ mbar, $\left.190{ }^{\circ} \mathrm{C}\right)$ to give a yield of $85 \%(0.266 \mathrm{~g},>95 \%$ by GC-MS) of pure $\mathbf{5 b}$.

\section{$N, N$-Dimethyl-2-[(2-phenyl-2-(4-N,N-dimetylaminophenyl))ethenyloxy]ethanamine (6a)}

Yellowish oil, $41 \%$ yield $\left(0.083 \mathrm{~g}\right.$, reaction at $180{ }^{\circ} \mathrm{C}, 55 \mathrm{~min},>95 \%$ by GC-MS). The compound was obtained as a $E / Z$ mixture. ${ }^{1} \mathrm{H}$ NMR $\left(400 \mathrm{MHz}, \mathrm{CDCl}_{3}, E / Z=3 / 7\right) \delta E 7.35-7.32(\mathrm{~m}, 0.6 \mathrm{H}), 7.24-7.08$ $(\mathrm{m}, 5.8 \mathrm{H}), E 7.01(\mathrm{~d}, J=8.8 \mathrm{~Hz}, 0.6 \mathrm{H}), E 6.60(\mathrm{~d}, J=8.8,0.6 \mathrm{H}), Z 6.58(\mathrm{~d}, J=8.8,1.4 \mathrm{H}), E 6.33(\mathrm{~s}$, $0.3 \mathrm{H}), Z 6.29(\mathrm{~s}, 0.7 \mathrm{H}), Z 3.90(\mathrm{t}, J=6.2 \mathrm{~Hz}, 1.4 \mathrm{H}), E 3.88(\mathrm{t}, J=6.2 \mathrm{~Hz}, 0.6 \mathrm{H}), Z 2.84(\mathrm{~s}, 4.2 \mathrm{H}), E$ $2.83(\mathrm{~s}, 1.8 \mathrm{H}), Z 2.55(\mathrm{t}, J=6.2 \mathrm{~Hz}, 1.4 \mathrm{H}), E 2.53(\mathrm{t}, J=6.2 \mathrm{~Hz}, 0.6 \mathrm{H}), Z 2.20(\mathrm{~s}, 4.2 \mathrm{H}), E 2.18(\mathrm{~s}$, 1.8H). ${ }^{13} \mathrm{C}$ NMR $\left(100 \mathrm{MHz}, \mathrm{CDCl}_{3}\right) \delta 149.7,149.3,144.0,143.9,141.4,138.5,130.8,130.1,129.4$, $128.8,128.4,128.0,126.5,126.1,120.9,120.8,112.7,112.2,71.8,58.9,58.8,46.3,40.9,40.8$. Major isomer: (Z)- Second GC-MS peak. MS m/z (relative intensity 70 eV) 310 (27, M $^{+}$), 72 (100), 58 (19). Minor isomer: (E)- First GC-MS peak. MS m/z (relative intensity $70 \mathrm{eV)} 310\left(28, \mathrm{M}^{+}\right), 72$ (100), 58 (20). (E/Z)- Anal. Calcd for $\mathrm{C}_{20} \mathrm{H}_{26} \mathrm{~N}_{2} \mathrm{O}: \mathrm{C}, 77.38 ; \mathrm{H}, 8.44$. Found: C, 77.34; H, 8.57. 
The reaction and purification was performed according to the General Procedure for Non-Symmetric Diarylations. (Z)-6b was the last eluted of the two isomers of $\mathbf{6 b}$ on chromatography. After evaporation of solvent (Z)-6b was obtained in $21 \%$ yield $(0.040 \mathrm{~g},>95 \%$ by GC-MS, colorless oil). The $\beta, \beta-$ arylated product $(E)$-6b eluted first from the chromatographic column could not be separated from one of the $\alpha, \beta$-arylated byproducts. The contaminated $(E)-\mathbf{6 b}$ was thus diluted with toluene $(10 \mathrm{~mL})$ and stirred vigorously for 6 minutes with concentrated $\mathrm{HCl}(20 \mathrm{~mL})$ at a temperature slightly below $0{ }^{\circ} \mathrm{C}$. The two-phase mixture was diluted with ice cold water and neutralized with sodium hydroxide during constant cooling. The organic phase was separated and the aqueous phase was additionally extracted twice with toluene. The combined organic phases was dried with $\mathrm{K}_{2} \mathrm{CO}_{3}$ (s), evaporated and further chromatographed to give $(E)-6 \mathbf{b}$ in $4 \%$ yield $(7.7 \mathrm{mg},>95 \%$ by GC-MS, colorless oil). Major isomer: (Z)- Last eluted $\beta, \beta$-arylated product on column chromatography, first eluted $\beta, \beta$-arylated product on GC-MS. ${ }^{1} \mathrm{H}$ NMR $\left(400 \mathrm{MHz}, \mathrm{CDCl}_{3}\right) \delta$ 7.16-7.13 (m, 3H), 7.07-7.03 (m, 2H), 7.02-6.98 (m, 3H), 6.73 $(\mathrm{s}, 1 \mathrm{H}), 3.88(\mathrm{t}, J=6.3 \mathrm{~Hz}, 2 \mathrm{H}), 2.44(\mathrm{t}, J=6.3 \mathrm{~Hz}, 2 \mathrm{H}), 2.12(\mathrm{~s}, 6 \mathrm{H}), 2.06(\mathrm{~s}, 6 \mathrm{H}) .{ }^{13} \mathrm{C} \mathrm{NMR}(100$ $\left.\mathrm{MHz}, \mathrm{CDCl}_{3}\right) \delta 144.6,139.0,137.4,136.7,128.8,127.5,127.3,126.1,125.2,118.3,71.6,59.1,46.3$, 20.4. MS m/z (relative intensity $70 \mathrm{eV}) 295\left(\mathrm{M}^{+}, 6\right), 72$ (100), 58 (70). Minor isomer: (E)- First eluted $\beta, \beta$-arylated product on column chromatography, second eluted $\beta, \beta$-arylated product on GC-MS. ${ }^{1} \mathrm{H}$ NMR (400 MHz, $\left.\mathrm{CDCl}_{3}\right) \delta$ 7.37-7.33 (m, 2H), 7.20-7.14 (m, 2H), 7.11-7.00 (m, 4H), 6.07 (s, $\left.1 \mathrm{H}\right), 3.96$ $(\mathrm{t}, J=6.0 \mathrm{~Hz}, 2 \mathrm{H}), 2.62(\mathrm{t}, J=6.0 \mathrm{~Hz}, 2 \mathrm{H}), 2.26(\mathrm{~s}, 6 \mathrm{H}), 2.11(\mathrm{~s}, 6 \mathrm{H}) .{ }^{13} \mathrm{C} \mathrm{NMR}\left(100 \mathrm{MHz}, \mathrm{CDCl}_{3}\right) \delta$ $146.3,139.1,137.2,128.33,128.26,127.7,127.4,126.2,116.0,72.2,59.2,46.5,20.7 . \mathrm{MS} \mathrm{m} / \mathrm{z}$ (relative intensity $70 \mathrm{eV}) 295\left(\mathrm{M}^{+}, 1\right), 72$ (100), 58 (55). (E/Z)- Anal. Calcd for $\mathrm{C}_{20} \mathrm{H}_{25} \mathrm{NO}: \mathrm{C}, 81.31 ; \mathrm{H}$, 8.53. Found: C, 81.2; H, 8.3.

\section{(E)-N,N-Dimethyl-2-[(2-phenyl-1-(2,6-dimethylphenyl))ethenyloxy]ethanamine}

Byproduct from the synthesis of $\mathbf{6 b}$, eluted before the two isomers of $\mathbf{6 b}$. Colorless oil, $4 \%$ yield $(7.7$ mg, $>95 \%$ by GC-MS). ${ }^{1} \mathrm{H}$ NMR (400 MHz, $\mathrm{CDCl}_{3}$ ) $\delta$ 7.78-7.72 (m, 2H), 7.38-7.32 (m, 2H), 7.22-7.16 
$(\mathrm{m}, 2 \mathrm{H}), 7.12-7.08(\mathrm{~m}, 2 \mathrm{H}), 5.26(\mathrm{~s}, 1 \mathrm{H}), 3.51(\mathrm{t}, J=6.2 \mathrm{~Hz}, 2 \mathrm{H}), 2.54(\mathrm{t}, J=6.2 \mathrm{~Hz}, 2 \mathrm{H}), 2.28(\mathrm{~s}$,

6H), $2.16(\mathrm{~s}, 6 \mathrm{H}) .{ }^{13} \mathrm{C}$ NMR $\left(100 \mathrm{MHz}, \mathrm{CDCl}_{3}\right) \delta 153.1,137.7,136.9,135.9,128.7,128.6,128.5$, 127.7, 125.9, 109.5, 66.7, 59.3, 46.3, 20.6. MS m/z (relative intensity 70 eV) 72 (100), 58 (55). Anal. Calcd for $\mathrm{C}_{20} \mathrm{H}_{25} \mathrm{NO}$ : C, 81.31; H, 8.53. Found: C, 81.1; H, 8.4.

\section{$N, N$-Dimethyl-2-[(2-phenyl-2-(o-cyanophenyl))ethenyloxy]ethanamine (6e)}

Yellowish oil, $47 \%$ yield $\left(0.089 \mathrm{~g}\right.$, reaction at $180{ }^{\circ} \mathrm{C}, 120 \mathrm{~min},>95 \%$ by GC-MS). Major isomer: $(Z)-$ First GC-MS peak, second eluted on column chromatography. FTIR (thin film): $2225,1636 \mathrm{~cm}^{-1} .{ }^{1} \mathrm{H}$ $\operatorname{NMR}\left(400 \mathrm{MHz}, \mathrm{CDCl}_{3}\right) \delta$ 7.60-7.56 (m, 1H), 7.44-7.38 (m, 1H), 7.25-7.06 (m, 5H), 7.04-6.98 (m, 2H), $6.69(\mathrm{~s}, 1 \mathrm{H}), 3.98(\mathrm{t}, J=6.0 \mathrm{~Hz}, 2 \mathrm{H}), 2.51(\mathrm{t}, J=6.0 \mathrm{~Hz}, 2 \mathrm{H}), 2.14(\mathrm{~s}, 6 \mathrm{H}) .{ }^{13} \mathrm{C} \mathrm{NMR}(100 \mathrm{MHz}$, $\left.\mathrm{CDCl}_{3}\right) \delta 145.6,141.0,137.8,131.9,131.4,130.5,127.5,126.2,125.8,125.6,117.7,116.5,112.9$, 70.4, 57.4, 44.8. MS m/z (relative intensity $70 \mathrm{eV}) 292\left(\mathrm{M}^{+}, 13\right), 72(62), 58$ (100). Minor isomer: $(E)-$ Second GC-MS peak, first eluted on chromatography. FTIR (thin film) 2224, $1694 \mathrm{~cm}^{-1} .{ }^{1} \mathrm{H}$ NMR (400 $\left.\mathrm{MHz}, \mathrm{CDCl}_{3}\right) \delta$ 7.61-7.58 (m, 1H), 7.45-7.41 (m, 1H), 7.31-7.12 (m, 7H), $6.48(\mathrm{~s}, 1 \mathrm{H}), 4.04(\mathrm{t}, J=6.0$ $\mathrm{Hz}, 2 \mathrm{H}), 2.63(\mathrm{t}, J=6.0 \mathrm{~Hz}, 2 \mathrm{H}), 2.24(\mathrm{~s}, 6 \mathrm{H}) .{ }^{13} \mathrm{C} \mathrm{NMR}\left(100 \mathrm{MHz}, \mathrm{CDCl}_{3}\right) \delta 148.2,144.7,137.1$, $133.8,132.7,132.0,129.6,128.3,127.4,127.1,119.0,117.2,113.4,72.5,58.9,46.3 . \mathrm{MS} \mathrm{m} / \mathrm{z}$ (relative intensity $70 \mathrm{eV}) 292\left(\mathrm{M}^{+}, 12\right), 72$ (49), 58 (100). (E/Z)- Anal. Calcd for $\mathrm{C}_{19} \mathrm{H}_{20} \mathrm{~N}_{2} \mathrm{O}: \mathrm{C}, 78.05 ; \mathrm{H}, 6.89$. Found: C, 78.05; H, 6.85 .

\section{$N, N$-Dimethyl-2-[(2-phenyl-2-(3-tienyl))ethenyloxy]ethanamine (6f)}

Before chromatography, small traces of 5a were removed by evaporation at $100{ }^{\circ} \mathrm{C}, 35$ mbar for 30 minutes. After column chromatography a colorless oil was obtained, $49 \%$ yield $(0.087 \mathrm{~g}$, reaction at $180{ }^{\circ} \mathrm{C}, 120 \mathrm{~min},>95 \%$ by GC-MS). Major isomer: (Z)- Second GC-MS peak, first eluted on chromatography. ${ }^{1} \mathrm{H}$ NMR $\left(400 \mathrm{MHz}, \mathrm{CDCl}_{3}\right) \delta$ 7.28-7.15 (m, 8H), $6.26(\mathrm{~s}, 1 \mathrm{H}), 3.99(\mathrm{t}, J=6.0 \mathrm{~Hz}$, 2H), $2.61(\mathrm{t}, J=6.0 \mathrm{~Hz}, 2 \mathrm{H}), 2.25(\mathrm{~s}, 6 \mathrm{H}) .{ }^{13} \mathrm{C} \mathrm{NMR}\left(100 \mathrm{MHz}, \mathrm{CDCl}_{3}\right) \delta 145.2,140.3,138.2,129.2$, 129.0, 128.4, 126.9, 124.1, 123.4, 116.3, 72.0, 58.9, 46.3. MS m/z (relative intensity $70 \mathrm{eV}) 273\left(\mathrm{M}^{+}\right.$, 
13), 72 (100), 58 (69). Minor isomer: (E)- First GC-MS peak, second eluted on chromatography. ${ }^{1} \mathrm{H}$ $\operatorname{NMR}\left(400 \mathrm{MHz}, \mathrm{CDCl}_{3}\right) \delta$ 7.37-7.32 (m, 2H), 7.27-7.13 (m, 4H), 6.94-6.90 (m, 1H), 6.88-6.85 (m, 1H), $6.56(\mathrm{~s}, 1 \mathrm{H}), 3.93(\mathrm{t}, J=6.1 \mathrm{~Hz}, 2 \mathrm{H}), 2.55(\mathrm{t}, J=6.1 \mathrm{~Hz}, 2 \mathrm{H}), 2.21(\mathrm{~s}, 6 \mathrm{H}) .{ }^{13} \mathrm{C} \mathrm{NMR}(100 \mathrm{MHz}$, $\left.\mathrm{CDCl}_{3}\right) \delta 145.0,141.5,137.6,129.8,128.2,127.4,126.9,125.6,120.9,116.4,72.1,58.9,46.4 . \mathrm{MS}$ m/z (relative intensity $70 \mathrm{eV}) 273\left(\mathrm{M}^{+}, 12\right), 72(100), 58$ (71). (E/Z)- Anal. Calcd for $\mathrm{C}_{16} \mathrm{H}_{19} \mathrm{NOS}$ : C, 70.29; H, 7.00. Found: C, 70.20; H, 6.85 .

Procedure for Catalyst Evaluation in the Synthesis of Diarylated Products.

Except for the different investigated catalytic systems the reactions were performed according to the General Procedure for Non-Symmetric Diarylations (entries 1-4) or according to the General Procedure for Symmetric Diarylations (Table S1, entry 5) using naphthalene (60 mg) as internal standard. 
Table S1. Product Distribution in $\beta$-Arylation Employing Different Palladium Catalysts

\begin{tabular}{|c|c|c|c|c|c|c|c|c|c|c|}
\hline Entry & $\mathrm{ArBr}$ & Olefin & $\begin{array}{l}\text { Equiv } \\
\mathrm{ArBr}\end{array}$ & $\begin{array}{l}\text { Temp } \\
\left({ }^{\circ} \mathrm{C}\right)\end{array}$ & $\begin{array}{l}{ }^{a} \text { Time } \\
(\min )\end{array}$ & $\begin{array}{l}\text { Catalyst } \\
(\mathrm{mol} \%)\end{array}$ & Product & $\begin{array}{l}\text { Conversion } \\
\text { of } \mathbf{5 a}(\%)\end{array}$ & $\begin{array}{l}\alpha, \beta / \\
\beta, \beta^{b}\end{array}$ & $E / Z^{b}$ \\
\hline \multirow[t]{4}{*}{1} & $2 b$ & $5 a$ & 5 & 180 & 10 & $0.5 \% 3$ & $6 c$ & 100 & $4 / 96$ & 29/71 \\
\hline & & & & & & $1 \% \mathrm{Pd}(\mathrm{OAc})_{2}+2 \% \mathrm{PPh}_{3}$ & & 100 & $4 / 96$ & $26 / 74$ \\
\hline & & & & & & $1 \% \mathrm{Pd}(\mathrm{OAc})_{2}+2 \%(o \text {-tol })_{3} \mathrm{P}$ & & 79 & $6 / 94$ & $31 / 69$ \\
\hline & & & & & & $1 \% \operatorname{Pd}\left(\mathrm{PPh}_{3}\right)_{2} \mathrm{Cl}_{2}$ & & 29 & $6 / 94$ & $23 / 77$ \\
\hline \multirow[t]{4}{*}{2} & 2c & $5 a$ & 2 & 180 & 10 & $0.5 \% 3$ & 4c & 99 & $4 / 96$ & \\
\hline & & & & & & $1 \% \mathrm{Pd}(\mathrm{OAc})_{2}+2 \% \mathrm{PPh}_{3}$ & & 81 & $4 / 96$ & \\
\hline & & & & & & $1 \% \mathrm{Pd}(\mathrm{OAc})_{2}+2 \%(o-\text { tol })_{3} \mathrm{P}$ & & 88 & $4 / 96$ & \\
\hline & & & & & & $1 \% \mathrm{Pd}\left(\mathrm{PPh}_{3}\right)_{2} \mathrm{Cl}_{2}$ & & 99 & $2 / 98$ & \\
\hline \multirow[t]{4}{*}{3} & $2 \mathrm{~h}$ & $5 a$ & 2 & 180 & 10 & $0.5 \% 3$ & $6 d$ & 95 & $5 / 95$ & $40 / 60$ \\
\hline & & & & & & $1 \% \mathrm{Pd}(\mathrm{OAc})_{2}+2 \% \mathrm{PPh}_{3}$ & & 96 & $2 / 98$ & $38 / 62$ \\
\hline & & & & & & $1 \% \mathrm{Pd}(\mathrm{OAc})_{2}+2 \%(o-\text { tol })_{3} \mathrm{P}$ & & 95 & $5 / 95$ & $39 / 61$ \\
\hline & & & & & & $1 \% \mathrm{Pd}\left(\mathrm{PPh}_{3}\right)_{2} \mathrm{Cl}_{2}$ & & 99 & $5 / 95$ & $36 / 64$ \\
\hline \multirow[t]{4}{*}{4} & $2 \mathbf{i}$ & $5 a$ & 5 & 180 & 120 & $0.5 \% 3$ & $6 e$ & 90 & $3 / 97$ & $22 / 78$ \\
\hline & & & & & & $1 \% \mathrm{Pd}(\mathrm{OAc})_{2}+2 \% \mathrm{PPh}_{3}$ & & 100 & $2 / 98$ & $25 / 75$ \\
\hline & & & & & & $1 \% \mathrm{Pd}(\mathrm{OAc})_{2}+2 \%(o-\text { tol })_{3} \mathrm{P}$ & & 100 & $2 / 98$ & $22 / 78$ \\
\hline & & & & & & $1 \% \mathrm{Pd}\left(\mathrm{PPh}_{3}\right)_{2} \mathrm{Cl}_{2}$ & & 100 & $1 / 99$ & $23 / 77$ \\
\hline \multirow[t]{4}{*}{$5^{c}$} & 2c & 1 & 3 & 180 & 10 & $0.5 \% 3$ & 4c & $81^{d}$ & $7 / 93$ & \\
\hline & & & & & & $1 \% \mathrm{Pd}(\mathrm{OAc})_{2}+2 \% \mathrm{PPh}_{3}$ & & $79^{d}$ & $6 / 94$ & \\
\hline & & & & & & $1 \% \mathrm{Pd}(\mathrm{OAc})_{2}+2 \%(o-\text { tol })_{3} \mathrm{P}$ & & $85^{d}$ & $8 / 92$ & \\
\hline & & & & & & $1 \% \mathrm{Pd}\left(\mathrm{PPh}_{3}\right)_{2} \mathrm{Cl}_{2}$ & & $77^{d}$ & $14 / 86$ & \\
\hline
\end{tabular}

${ }^{a}$ Single-mode microwave heating. ${ }^{b}$ Determined by GC-MS. Each value comprises an average of three runs. Conversion was determined using naphthalene as internal standard. ${ }^{c}$ Used General Procedure for Symetrical Arylations. ${ }^{d}$ Value is reflecting $\mathbf{5} \mathbf{a}$ as a quotient of used amount of $\mathbf{1}$.

\section{References}

(1) Nilsson, P.; Larhed, M.; Hallberg, A. J. Am. Chem. Soc. 2001, 123, 8217-8225.

(2) Andersson, C. M.; Larsson, J.; Hallberg, A. J. Org. Chem. 1990, 55, 5757-5761.

(3) Larhed, M.; Andersson, C. M.; Hallberg, A. Tetrahedron 1994, 50, 285-304.

(4) Cabri, W.; Candiani, I.; Bedeschi, A.; Santi, R. J. Org. Chem. 1990, 55, 3654-3655. 